\title{
Current issues and perspectives in herbal hepatotoxicity: a hidden epidemic
}

\author{
Fabio Firenzuoli $\cdot$ Luigi Gori $\cdot$ Alessandro Mugelli • \\ Alfredo Vannacci
}

Received: 23 July 2012/ Accepted: 15 September 2012/Published online: 13 October 2012

(C) SIMI 2012

Hepatic impairment resulting from the use of conventional drugs is widely acknowledged, yet there is a scant awareness of the potential hepatotoxicity of herbal medicines; nevertheless there is an increasing appeal of herbal medicines both for patients and health caregivers, only partially explained by the return to the use of natural products occurring along with a cultural ecological movement in all industrialized countries.

Some herbs are well known for their direct hepatotoxicity (Teucrium chamaedrys, Senecio vulgaris, Morinda citrifolia, Ephedra sinica, Mentha pulegium, plants containing antraquinones, etc.) or for drug interferences (Panax ginseng, Citrus paradisi, Piper nigrum, Silybum marianum, Camellia sinensis, Monascus ruber, etc.). For many herbs a sufficient knowledge of their pharmacodynamic and pharmacokinetic is lacking, this is particularly true when herbal products are used in combinations (as it often happens in Oriental traditional medicines), making it difficult to perform a causality assessment for the single herbs involved. As suggested in the article by Licata et al. [1] herbal hepatotoxicity may become a hidden epidemic.

Known herbal extracts with direct hepatotoxicity are very few, nevertheless an unexpected idiosyncratic reaction is always possible, but physicians who are not true experts in herbal medicine actually seem to overlook this important toxicological topic. This is similar with other important problems (herbal-drug interference, side effects, long term toxicity) that consumers and health caregivers (including herbalists, naturopaths, dieticians, gym trainers,

F. Firenzuoli $(\bowtie) \cdot$ L. Gori · A. Mugelli · A. Vannacci Department of Preclinical and Clinical Pharmacology, Center for Integrative Medicine, University of Florence, Viale G. Pieraccini 6, 50139 Florence, Italy

e-mail: fabio.firenzuoli@unifi.it cosmeticians, masseurs, et al.) often ignore, thinking that herb is synonymous with safety. We are completely in agreement with Licata et al. [1] that the safety of herbal products must be evaluated on the basis of existing scientific literature. In fact what is it that differentiates an herbal product from a conventional drug? A drug is considered safe and effective only if its preparation is standardized, and its safety and efficacy definitely assessed. Unfortunately, few of the used herbs are standardized by methods that can ensure a consistent product [1].

Currently the main important and fast growing issue is herb-drug interactions: who can really estimate the real hepatotoxic risk of combining paracetamol with herbs that can interact with its metabolism? [2]. Because of their biological defence function, plant secondary metabolites act by targeting and disrupting the cell membrane, by binding and inhibiting specific proteins, or they adhere to or intercalate into RNA or DNA. Phytotherapeutics may exhibit pharmacological effects by the synergistic or antagonistic interaction of many phytochemicals. Mechanistic reasons for interactions are bioavailability, interference with cellular transport processes, activation of pro-drugs or deactivation of active compounds to inactive metabolites, action of synergistic partners at different points of the same signalling cascade (multi-target effects) or inhibition of binding to target proteins. [3]. All of these are important clues whose definite biological effects are impossible to control entirely.

Another significant topic is the correct preparation of an extract: it is very important to prescribe herbal extracts undergoing a correct chain of controls in pharmacies or industrial manufactures, avoiding use of self prepared remedies, or worse, bought on the internet directly from "natural merchandisers," which may skip any hygienic or authority control [4]. 
Rigid controls of vegetal derivatives are of paramount importance because herbs may be corrupted by aflatoxins [5] (or other natural toxic substances), heavy metals (lead, chromium, cadmium, etc.), or adulterated with undeclared herbs or even with synthetic drugs [6].

Another relevant issue is the use of herbal remedies in fragile populations such as pregnant women, and children in neonatal or paediatric age, by patients or parents passionate for a sort of "ecological medicine", convinced that natural is always safe [7]. Even many cancer patients trust herbal remedies, using them almost always without any medical advice, and threaten the success of conventional medicine, and jeopardize an effective treatment by acting on false hopes on herbal remedies that can severely interfere with effective oncological therapies.

The use of herbs as an adjunct to weight control diets in human beings and contemporary fasting reduces hepatic glutathione, enhancing metabolite-mediated hepatotoxicity. Glutathione is a major antioxidant as well as redox and cell signalling regulator in liver. Glutathione guards cells against oxidative injury by reducing $\mathrm{H}_{2} \mathrm{O}_{2}$ and scavenging reactive oxygen and nitrogen radicals. In addition, glutathione-induced redox shift with or without ROS subjects some cellular proteins to varied forms of oxidation, impairing the function of signal transduction and transcription factor molecules [8].

Since many herbal products on the market have not been thoroughly tested for their pharmacology and toxicology, pharmacovigilance is of paramount importance in detecting unwanted reactions [5, 9], because clinical patterns and severity can be highly variable, even for the same product [1].

This issue has become very important for herbal toxicogenomics. Changes in transcriptomics, proteomics or metabolite profiles may serve as early, sensitive indicators of a potential toxicity, and are thought to precede toxic outcomes; in particular, gene expression data (transcriptomics) are thought to be more sensitive than traditional toxicological endpoints $[5,10]$. Toxicogenomics can also help to bridge traditional in vitro and in vivo toxicity assessment, and might be a very effective tool in studying the safety of herbal medicines by expanding the predictivity of potential toxicity in earlier stages of product development $[5,10]$.

In addition, the inhibition or induction of cytochrome by herbal constituents may decrease or increase the formation of toxic drug metabolites, and thus inhibit or promote carcinogenesis, as cytochromes play an important role in pro-carcinogen activation. Due to the wide use and easy availability of herbal medicines, further research should be conducted to ensure the safety and quality of herbal medicine and their biological action on drug metabolites.

Herbal medicines in Europe come from all traditions including Chinese, Indian, North and South American and
African systems as well as that of European systems. This diversity adds to the challenges of herbal pharmacovigilance, including basic questions such as defining the most appropriate herb naming system (botanical, common, pharmaceutical name or herbal drug name) and validation of the botanical identity of the herbal ingredients [9]. Although some modifications may be needed, developing separate systems for herbals is not the answer, as this is likely to add complications and cause confusion if different forms or systems are used with the risk of reducing reporting rates further [11]. Unfortunately a botanically correct label does not necessarily state the product actually contains what is listed on the label [8]. In cases of serious adverse reactions where specific toxins are suspected, then laboratory analysis of the product/herb may be the only reliable tool to definitely verify the reports.

Drug-induced liver injury represents the principal cause of acute liver failure and orthotopic liver transplantation in Western countries, and the interference due to herb and their derivatives may be very difficult to identify, especially in patients using many different drugs, a common toxicological topic in Western countries [12]. A very large number of different drugs and medicinal herbs have been associated with liver injury, but in just a few of them, we know the exact process that causes liver disease. Moreover, a final diagnosis is very difficult because a specific biomarker of damage is absent, and the clinical picture is common to other liver diseases [12].

Regulations and pharmacovigilance regarding herbal drugs are still incomplete, and need to be improved, and is alike important to spread correct education and information:

1. To physicians, and allied health caregivers especially nurses and midwives, who often underestimate the risk of natural products. Almost never in emergency, obstetrical or surgical departments are patients routinely queried about use of herbal remedies. While would be very important to know the risks and epidemiology to sharpen the technique of anamnesis and improve pharmacovigilance reports.

2. To all health caregivers physicians, herbalists, naturopaths, and others who counsel and prescribe medicinal herbs so that they refer only to scientific medicine sources and not to patent medicines and systems based on hopes or wish fulfillment fantasy.

3. To patients, considering that $40 \%$ of the people in UK think herbal medicines are safe because they are natural (Ipsos MORI/MHRA, survey 2008), so that they do not entrust their health to self-medicine, "internet medicine", ancient medicine or trusting alternative medicine just because they wish it to be a safe alternative to scientific medicine. 
Finally concerning the new legislation of the EU [13] with the transition from detailed description of the Pharmacovigilance System to the Pharmacovigilance System Master File, that introduces the principle of EU single assessment of periodic safety update reports (PSURs), where a substance is authorized in more than one Member State and establishes the list of European Union Reference Dates (EURD). The principle of EU single assessment of periodic safety update reports (PSURs) will not be required for traditional medicinal products authorized under the articles of Directive 2001/83/EC (homeopathic medicines and traditional herbal medicine) [13], we think should be reconsidered. We believe that in terms of toxicity assessment, these products should be considered as if they were conventional drugs, in order to warrant the highest possible level of safety for patients assuming all the different kinds of 'natural drugs'.

Finally, physician and patient awareness remains central to the early identification of herbal toxicity, and must be improved for the optimal use of these agents [1].

Clinicians should always investigate the consumption of herbal products during anamnesis, and check that dose recommendations are not exceeded [1]. As practiced for conventional medicine, patients should be advised of possible adverse effects, and encouraged to report them as soon as possible, even if non-specific in nature [1] and educated that thinking natural is synonymous with safe is a misconception.

Conflict of interest None.

\section{References}

1. Licata A, Macaluso FS, Craxì A (2012) Herbal hepatotoxicity: a hidden epidemic. Intern Emerg Med. doi:10.1007/s11739-0120777-x
2. Zhao L, Pickering G (2011) Paracetamol metabolism and related genetic differences. Drug Metab Rev 43:41-52

3. Efferth T, Koch E (2011) Complex interactions between phytochemicals. The multi-target therapeutic concept of phytotherapy. Curr Drug Targets 12:122-132

4. Firenzuoli F, Gori L, Di Simone L, Morsuillo M (2006) Herbs on the internet: risky information. Recenti Prog Med 97:189-192

5. Firenzuoli F, Gori L (2007) Herbal medicine today: clinical and research issues. Evid Based Complement Alternat Med. 4(Suppl 1):37-40

6. Vannacci A, Lapi F, Baronti R, Gallo E, Gori L, Mugelli A, Firenzuoli F (2009) Too much effectiveness from a herbal drug. Br J Clin Pharmacol 67:473-474

7. Lapi F, Vannacci A, Moschini M, Cipollini F, Gallo E, Banchelli G, Cecchi E, Di Pirro M, Giovannini MG, Cariglia MT, Gori L, Firenzuoli F, Mugelli A (2010) Use, attitudes and knowledge of complementary and alternative drugs (CADs) among pregnant women: a preliminary survey in Tuscany. Evid Based Complement Alternat Med. 7(4):477-486

8. Yuan L, Kaplowitz N (2009) Glutathione in liver diseases and hepatotoxicity. Mol Aspects Med 30:29-41

9. Shaw D, Graeme L, Pierre D, Elizabeth W, Kelvin C (2012) Pharmacovigilance of herbal medicine. J Ethnopharmacol 140: 513-518

10. Ouedraogo M, Baudoux T, Stévigny C, Nortier J, Colet JM, Efferth T, Qu F, Zhou J, Chan K, Shaw D, Pelkonen O, Duez P (2012) Review of current and "omics" methods for assessing the toxicity (genotoxicity, teratogenicity and nephrotoxicity) of herbal medicines and mushrooms. J Ethnopharmacol 140:492-512

11. Menniti-Ippolito F, Mazzanti G, Santuccio C, Moro P, Calapai G, Firenzuoli F, Valeri F, Raschetti R (2008) Surveillance of suspected adverse reactions to natural health products in Italy. Pharmacoepidemiol Drug Saf 17:626-635

12. Abenavoli L, Libri E, Bosco D, Gallo D, Luzza F (2012) Druginduced liver injury. Recenti Prog Med 103:79-84

13. MHRA. 2012 pharmacovigilance legislation changes: implications for marketing authorisation holders. http://www.mhra.gov. uk/Howweregulate/Medicines/Pharmacovigilancelegislation/2012 pharmainfoformahs/index.htm\#l1. Accessed on 12th july 2012 